(C) [2009] IEEE. Reprinted, with permission, from Guo, Liuming; Guo, Ningning; Wang, Shuhong; Qiu, Jie; Zhu, Jianguo; Guo, Youguang; Wang, Yi. 2009, Optimization for capacitor-driven coilgun based on equivalent circuit model and genetic algorithm', Proceedings of IEEE Energy Conversion Conference \& Exposition, pp. 234-239 20-24 Sept. 2009. This material is posted here with permission of the IEEE. Such permission of the IEEE does not in any way imply IEEE endorsement of any of the University of Technology, Sydney's products or services. Internal or personal use of this material is permitted. However, permission to reprint/republish this material for advertising or promotional purposes or for creating new collective works for resale or redistribution must be obtained from the IEEE by writing to pubs-permissions@ieee.org. By choosing to view this document, you agree to all provisions of the copyright laws protecting it. 


\section{Optimization for Capacitor-Driven Coilgun Based on Equivalent Circuit Model and Genetic Algorithm}

\author{
Liuming Guo, Ningning Guo, \\ Shuhong Wang, Jie Qiu \\ Faculty of Electrical Engineering \\ Xi'an Jiaotong University \\ 28 West Xianning Road \\ Xi'an, 710049, CHINA \\ shwang@mail.xjtu.edu.cn
}

\author{
Jian Guo Zhu*, Youguang Guo*, Yi Wang \\ *IEEE Senior Member \\ School of Electrical, Mechanical and Mechatronic Systems \\ University of Technology, Sydney \\ 1 Broadway \\ NSW 2007, Australia \\ joe@eng.uts.edu.au
}

\begin{abstract}
This paper presents an analysis and optimal design of a capacitor-driven inductive coilgun. The electromagnetic FEA model of the capacitor-driven inductance coilgun is established and simulated by using Ansoft. In order to speed up the optimization, an equivalent circuit is designed for simulation of the performances of coilgun. A piece-wise model of projectile is built to account for the eddy current. The simulated results are compared with the modeling of FEA value to verify the developed circuit model. It shows that the results are in a good agreement. The optimization of coilgun is achieved by employing the genetic algorithm (GA) based on the circuit model. It is suggested that the proposed algorithm achieves a better result in the energy efficiency of the coilgun system, and obtain a higher muzzle velocity of the projectile.
\end{abstract}

Index Terms--Capacitor-driven inductive coilgun, Circuit model, Treanor method, Genetic algorithm (GA).

\section{INTRODUCTION}

Electromagnetic launch systems have advantages compared with the existing chemical launch systems. Generally the electromagnetic launchers are categorized into three kinds of systems: railguns, coilguns and reconnection guns. The railgun is suitable for small projectiles and is conceptually simple. However, it has inherent problems and limitations. The coilgun may be applied in large projectile launching. Especially, the capacitor-driven inductive coilgun, which is almost free from the friction between barrel and projectile, is recommended for rapid acceleration. It can be easily installed and repeatedly used, the electromagnetic launch does not damage the launch devices, and the force exerted on the projectile is distributed uniformly [1]. The reconnection gun's potential performance is shown to be superior to that of a modern railgun for projectiles with a mass of greater than a few hundred grams. It has several advantages for producing higher acceleration including no barrel, no drop in acceleration with increase of projectile mass, higher peak pressure on the projectile and smaller differences between average and peak pressure [2].

The analysis of inductive coilgun is relatively complex. It is not easy to design the coilgun based on an analytical approach due to time-varying mutual inductances between the driver coils and the projectile in the transient launch process.
Hence it is necessary to rely on finite element software or numerical approaches to design a coilgun to achieve good launch characteristics. The design using the numerical approaches usually requires the system modeling technique as well as optimization algorithm [3]-[5].

The capacitor-driven coilguns are categorized into two kinds: the "asynchronous" mode and the "synchronous" mode [3]. In the first case, the driver coils may be connected in series or in parallel to form a limited number of phase windings, as is done in conventional machines. The driver coils on the barrel are energized with a certain time sequence so as to generate a traveling electromagnetic wave, which interact with the current in the projectile. The characteristic of this mode is that the performance is relatively poor, because relative movement between projectile and traveling wave causes the induced current reduced. In the second case, the velocity of the projectile is exactly equal to that of the traveling wave. The currents must be impressed on the projectile by external circuit. The driver coils must be energized separately by external source, which is controlled by switches. The turn on and off time of the switches must be governed by the position of the projectile. The size of each capacitor must be chosen considering the speed of projectile and derivative of mutual inductance with respect to displacement. Because of the complexity of the control system and the power supply system, it is not easy to achieve a good performance.

In this paper, an optimal design of capacitor-driven inductive coilgun by using a deduced equivalent circuit model and genetic algorithm (GA) is presented. To deduce the time of optimization, an equivalent circuit model coupled with the motion equation of the projectile is built up for the performance simulation of the coilgun. The 2-Dimensional axial-symmetrical transient finite element analysis (FEA) and the equivalent circuit model are respectively applied to simulation of a prototype designed by Sandia Laboratory [6][7]. Comparison of the simulated results calculated by both FEA and circuit model and the experimental results provided by Sandia laboratory shows the correction of circuit model. Concerning the optimization problem of the coilgun, the genetic algorithm is applied to the design parameter 
optimization of one-stage coilgun, which demonstrates the potential of the proposed design method.

\section{EQuivalent CIRCUIT MOdel}

Fig. 1 shows the structure of a capacitor-driven inductive coilgun [8]. The coilgun consists of a moving projectile and driver coils fixed on the barrel. The capacitor bank is connected to the coils as an energy source. The switches $S_{1}$ to $\mathrm{S}_{\mathrm{n}}$ are turned on and off in controlled sequence. The transient magnetic field is established inside the barrel, and eddy currents are induced on the surface of the conductive projectiles. The projectile is accelerated by the electromagnetic force produced by the interaction between the traveling magnetic field and the eddy currents in the projectile.

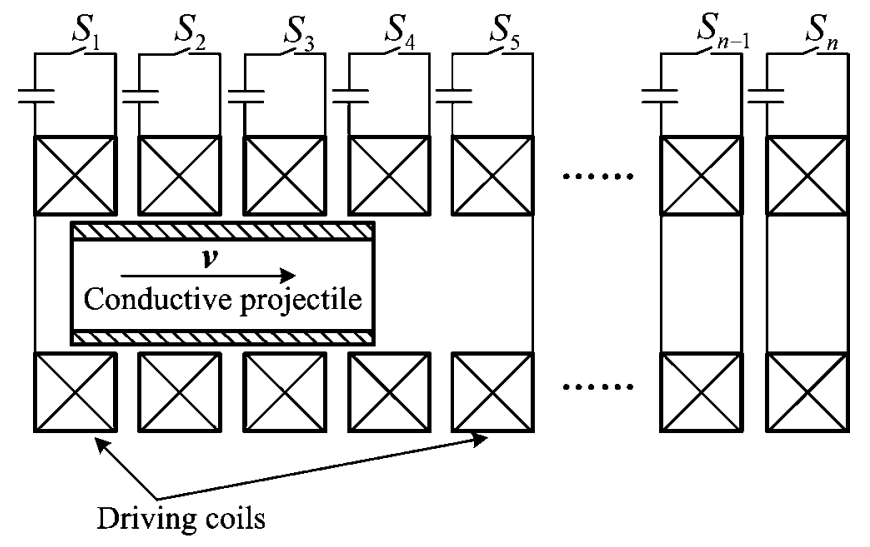

Fig. 1. Structure of a capacitor-driven coilgun

Since the distribution of eddy currents in the projectile is not uniform, as shown in Fig. 2(a), the projectile is assumed to be composed of shorted coils. Fig. 2(b) shows the geometry of the armature, i.e. projectile.

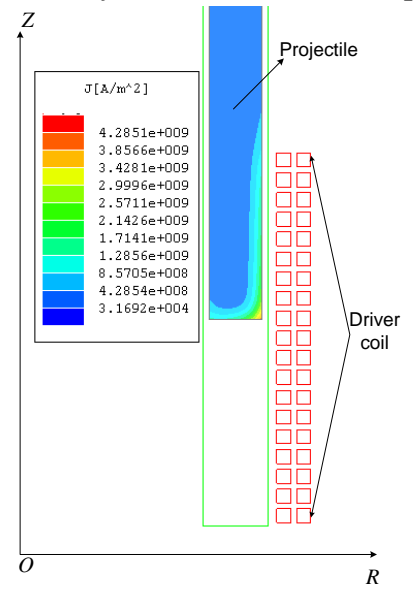

(a) Current density distribution in projectile

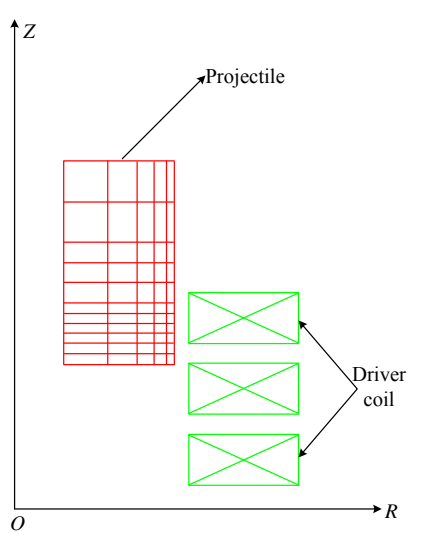

(b) Equivalent projectile model
Fig. 2. Equivalent projectile model
In this paper, an improved projectile model is presented. The armature is approximated as a collection of rectangular cross-section hoops, whose number and geometry are determined to achieve sufficient precision of the current density distribution. The current density is uniform in any hoop. The induced currents flowing through different hoops are also different. The multi-turn coils are modeled as single element because their current density is uniform across the face. The equivalent circuit for this system is shown in Fig.3. The inductances in the equivalent circuit are calculated by using the Flux Integration method.

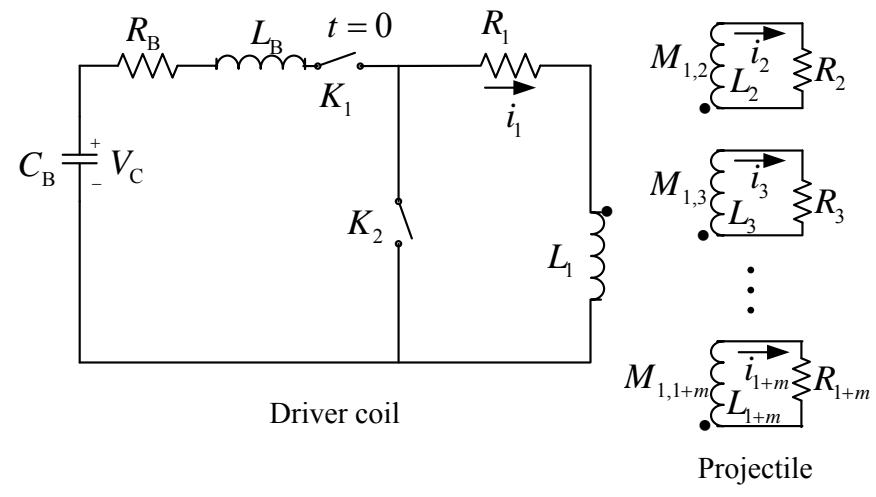

Fig. 3. Equivalent circuit model of a capacitor-driven coilgun

The circuit equations and the equation of motion of the projectile can be seen as follows[3].

$$
\left\{\begin{array}{l}
\{[L]+[M]\} \frac{d[I]}{d t}=\left[V_{C}\right]-[R] \cdot[I]-v\left[\frac{d M}{d x}\right] \cdot[I] \\
{[C] \frac{d\left[V_{C}\right]}{d t}=-\left[I_{d}\right]} \\
M_{P} \frac{d v}{d t}=\sum_{p=1}^{m} \sum_{d=1}^{n} I_{p} I_{d} \frac{d M_{p d}}{d x} \\
\frac{d x}{d t}=v
\end{array}\right.
$$

where $L$ and $M$ are self- and mutual inductances matrices of driver coils and projectile, respectively. $V_{\mathrm{c}}$ is the voltage of capacity bank. $I$ is a vector of currents flowed in driver coil and hoops in projectile. $M_{\mathrm{p}} 、 v$ and $X$ are the mass velocity and position of the projectile, respectively. Equ. (1) is solved by using the Treanor method [9].

\section{COMPARISON AMONG SIMULATION AND EXPERIMENT RESULTS}

To verify the validity of the simulation using improved projectile model and equivalent circuit model, the numerical results are compared with the FEA results and the experimental results for the prototype of one-stage coilgun produced by Sandia Laboratory, USA. The specifications of the prototype are listed in Table I. The model of the coilgun in Maxwell 2D, a commercial FEA package, is the same as 
that in Fig. 2(a). The initial velocity of projectile is $11.9 \mathrm{~m} / \mathrm{s}$.

TABLE I

\begin{tabular}{|c|c|}
\hline design variables & Values \\
\hline Coil turns of driver coils & 38 \\
\hline Thickness of driver coils (mm) & 11.3 \\
\hline Length of driver coils (mm) & 107 \\
\hline Inner diameter of driver coils (mm) & 147 \\
\hline Length of projectile (mm) & 203 \\
\hline Thickness of projectile (mm) & 15 \\
\hline Outer diameter of projectile $(\mathrm{mm})$ & 140 \\
\hline Voltage (V) & 4600 \\
\hline Capacitance (mF) & 20.485 \\
\hline Initial position of projectile $(\mathrm{mm})$ & 28.5 \\
\hline Material of driver coils & Copper \\
\hline Material of projectile & Aluminum \\
\hline Mass of projectile $(\mathrm{kg})$ & 5.0 \\
\hline
\end{tabular}

Simulated results by FEA and the developed circuit model are shown in Figs. 4 to 7 respectively. The velocity of projectile at the end of stage one and the peak current of the driver coil by FEA are $87.83 \mathrm{~m} / \mathrm{s}$ and $37.153 \mathrm{kA}$, respectively, while that by developed circuit model are $86.82 \mathrm{~m} / \mathrm{s}$ and $37.374 \mathrm{kA}$, respectively. It is noted that the calculations by the developed circuit model and the FEA are in a good agreement.

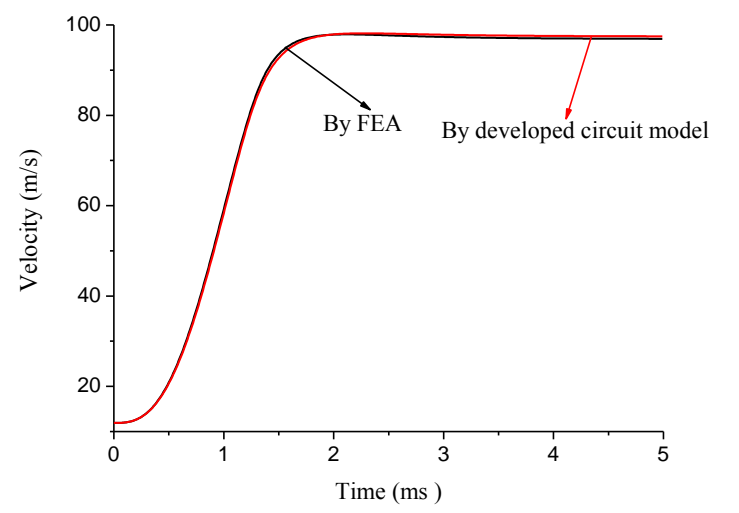

Fig. 4. Velocity calculated by FEA and the developed circuit model

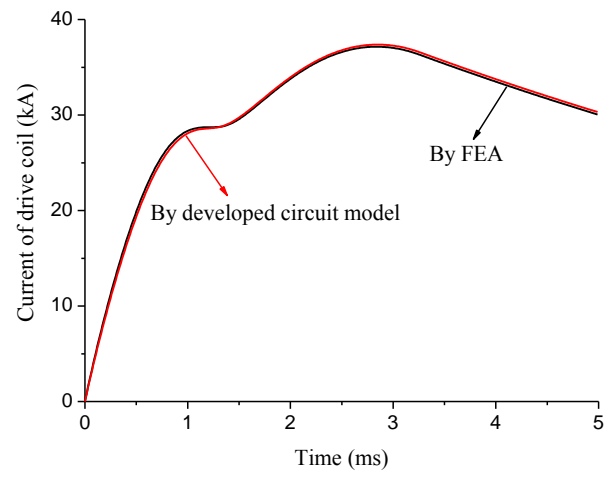

Fig. 5. Calculated current flowing in the coils

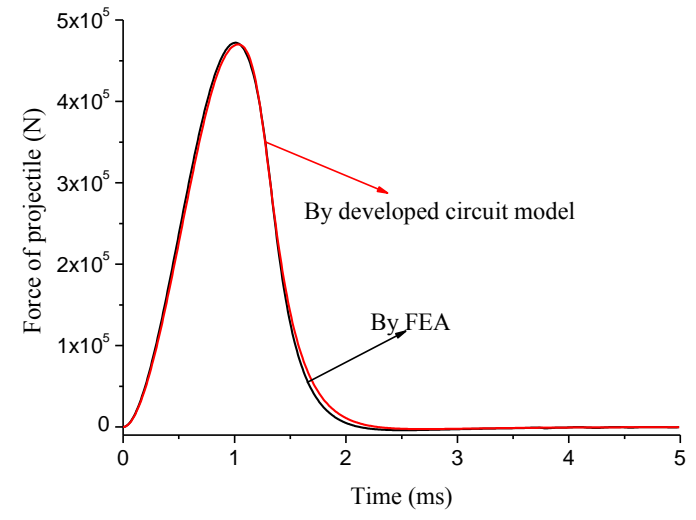

Fig. 6. Force of projectile in the Sandia model

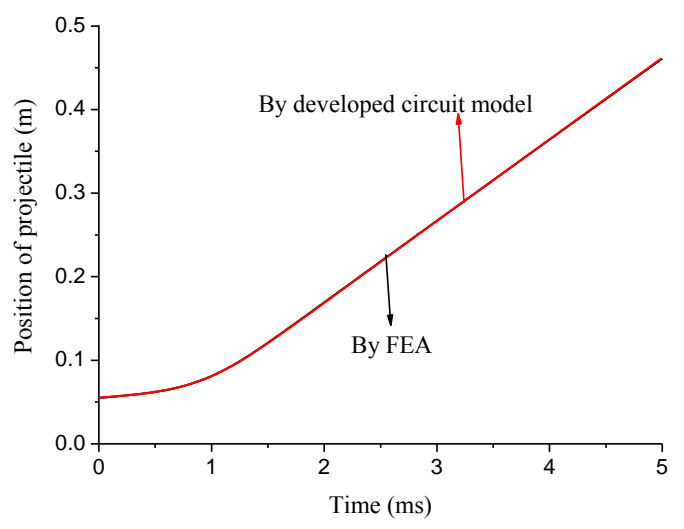

Fig. 7. Position of projectile in the Sandia model

In the experiment results provided by [7], the peak current of the driver coil and the muzzle velocity of projectile at the end of Stage One are $30.8 \mathrm{kA}$ and $80.0 \mathrm{~m} / \mathrm{s}$ respectively. Because the position at which the capacitor bank switch is triggered was not provided in [7], there is error between simulation and experiment results.

\section{INFLUENCE OF DESIGN PARAMETERS ON THE PROPERTIES OF INDUCTION ELECTROMAGNETIC LAUNCH SYSTEM}

In order to analyze the influence of various design parameters on the properties of the induction electromagnetic launch system, the first stage of the six-stage coilgun of Sandia Laboratory in the United States is taken as an example. Only one parameter is changed for each time to carry out simulation analysis to study the effect of the parameters.

\section{A. Influence of projectile length on electromagnetic launch system}

The changes in the length of projectile affects not only the muzzle velocity of projectile (short as velocity), but also the efficiency of the launch system. By changing the length of projectile, the speed and launch efficiency curves are shown in Fig. 8. As the length of projectile increases, the quality of the projectile increases while the velocity gradually decreases. 
The maximum launch efficiency exists because the induced current mainly concentrates in the rear of the projectile so that we can reduce the length of projectile appropriately and the efficiency will increase. However if the length of projectile is over-reduced, the current access will become smaller so that the induced currents will decline, resulting in reduced efficiency.

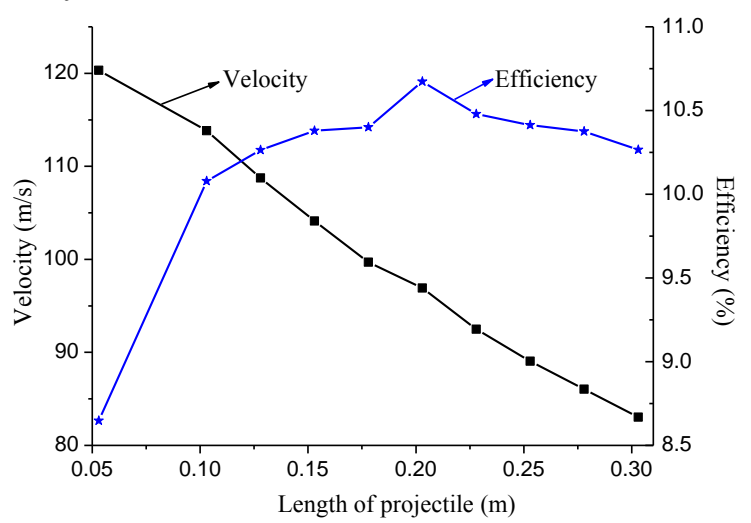

Fig. 8. Simulation results within various lengths of projectile

\section{B. Influence of thickness of projectile on electromagnetic launch system}

If the other conditions remain unchanged, e.g. the outer diameter of projectile is fixed at $70 \mathrm{~mm}$, a series of simulation are carried out with different thicknesses of the projectile, and simulation results are shown in Fig. 9. In the process of changing the thickness of projectile, the maximum velocity of launch system can be found. There is an optimum thickness of projectile to reach the highest efficiency.

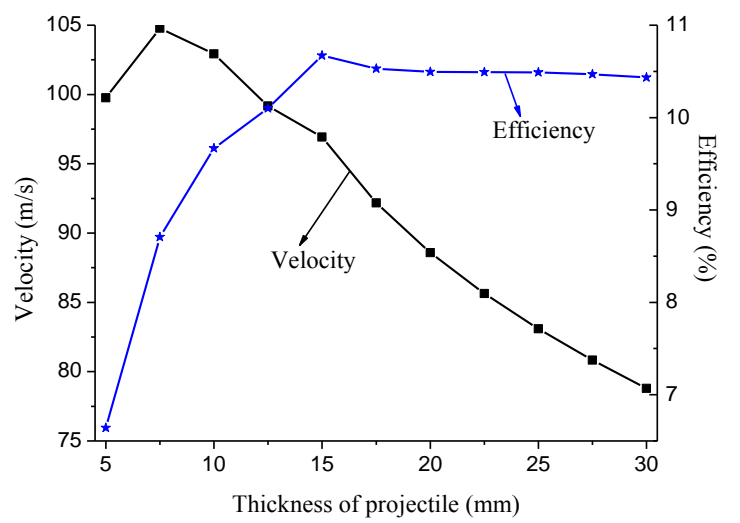

Fig. 9. Simulation results within various thicknesses of projectile

\section{Influence of outer semi-diameter of projectile on electromagnetic launch system}

The outer radius of projectile affects the magnetic coupling between the projectile and the coil. When the other conditions remain unchanged, simulation is carried out with different radius of the projectile, and the simulation results are shown in Fig. 10. Both the muzzle velocity and efficiency increase gradually when the outer radius of projectile increases, because the larger the outer diameter of projectile is, the more closely the projectile and the coil are magnetically coupled, resulting in the increase of the electromagnetic efficiency.

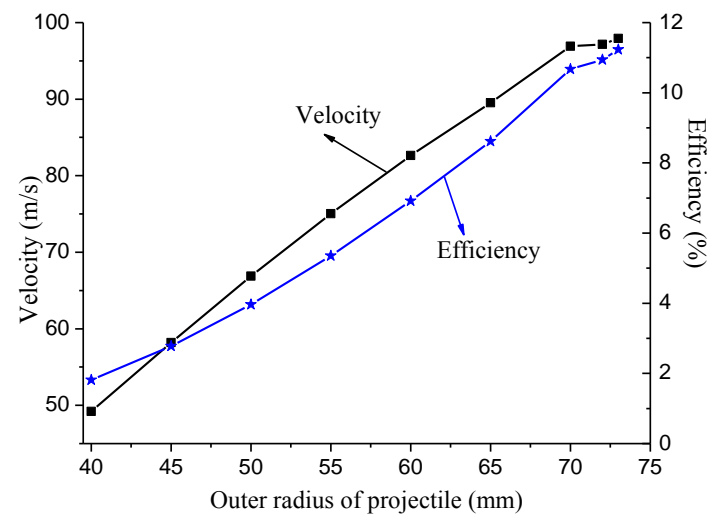

Fig. 10. Simulation within various outer radiuses of projectile

\section{Influence of number of coil turns on electromagnetic launch system}

The number of coil turns is an important impact factor on the velocity and efficiency of the launch system. When the other conditions remain unchanged, simulation is carried out with different numbers of coil turns and the velocity curves are shown in Fig. 11. It indicates that the number of coil turns in the driver coil design should be minimized, especially that in the width direction.

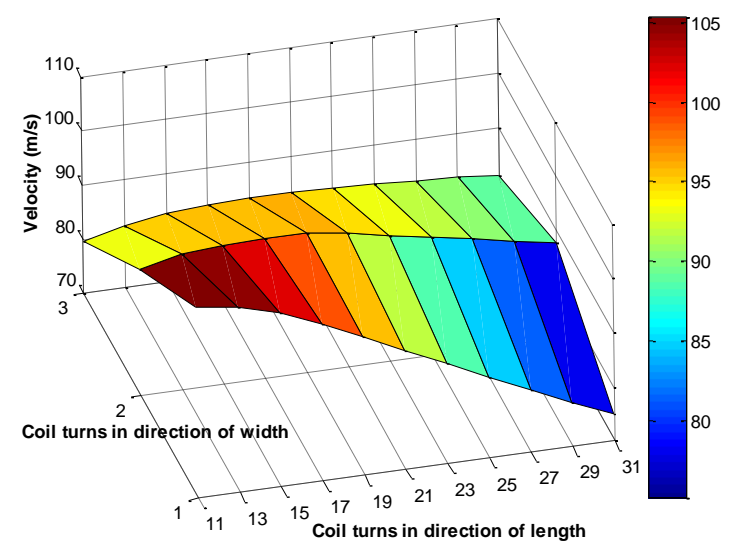

Fig. 11. Simulation results with various numbers of coil turns

\section{E. Influence of initial position of projectile on electromagnetic launch system}

According to the principle of electromagnetic launch system, the initial position of the projectile should be behind the center face of the coil. The initial position has great impact on the performance of the launch system. When the other conditions remain unchanged, simulation is carried out with different initial positions of the projectile, and the velocity curve changing with the center-distance of the driver coil is shown in Fig. 12. Therefore initial position of projectile should be properly chosen. 


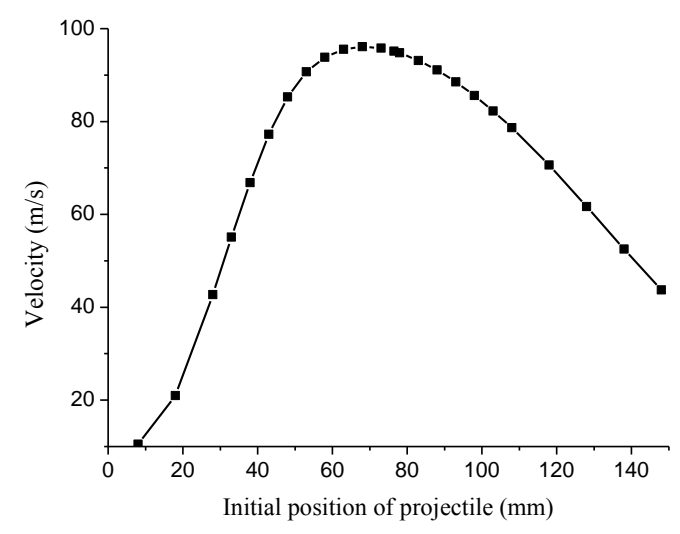

Fig. 12. Simulation results with various initial positions of projectile

\section{F. Influence of capacitance on electromagnetic launch system}

Simulation in the case of different discharges of capacitor is carried out, and the velocity curve changing with different capacitances is shown in Fig. 13. It indicates that the greater the capacitance is, the larger the muzzle velocity is obtained. There is an optimum capacitance value to reach the highest efficiency.

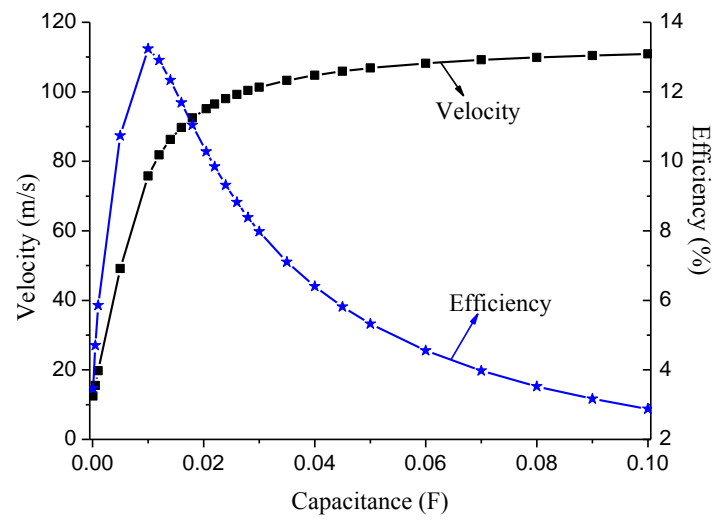

Fig. 13. Simulation results with various capacitances

\section{G. Influence of voltage of capacitor on electromagnetic launch system}

Simulation results in the case of different charge voltages of capacitor are shown in Fig. 14. The velocity curve, changing with the voltage of the capacitor, indicates that the higher the voltage of the capacitor is, the greater the muzzle velocity will be. There is an optimum charge voltage to reach the highest efficiency.

\section{OPTIMIZATION OF COILGUN}

The objective function is to achieve the maximum of velocity of projectile at the end of driver coil. The outer diameter, length and thickness of projectile, turns of coil, thickness of coil, charging voltage, capacitance and initial projectile position are chosen as the design variables.
According to the simulation results in part IV, the ranges of design variables are given in Table II.

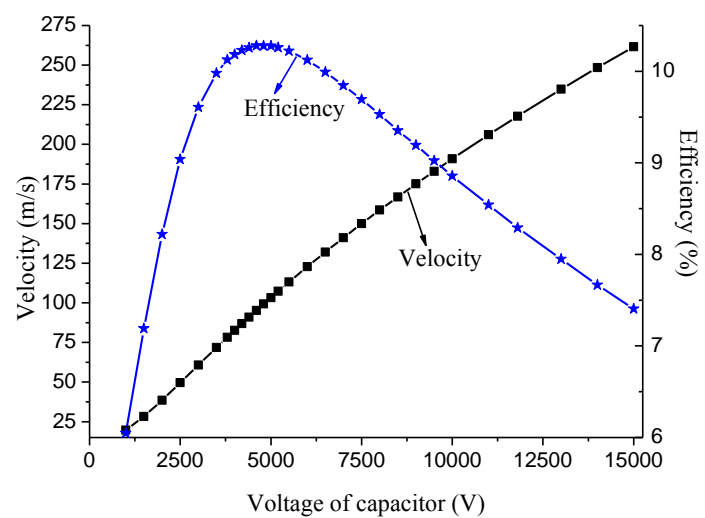

Fig. 14. Simulation results with various voltages of capacitor

TABLE II

OPTIMAL DESIGN FOR THE DRIVEN COILGUN

\begin{tabular}{lll}
\hline & $\begin{array}{l}\text { Optimal } \\
\text { results }\end{array}$ & $\begin{array}{l}\text { Constraints of design } \\
\text { variables }\end{array}$ \\
\hline Coil turns of driver coils & $11 \mathrm{turns}$ & {$[10 \sim 30]^{*}[1 \sim 5]$ turns } \\
Thickness of driver coils & $5.64 \mathrm{~mm}$ & $5.64 \sim 16.92 \mathrm{~mm}$ \\
Length of driver coils & $62 \mathrm{~mm}$ & $56.4 \sim 169.2 \mathrm{~mm}$ \\
Inner diameter of driver coils & $130 \mathrm{~mm}$ & $130 \sim 170 \mathrm{~mm}$ \\
Length of projectile & $286.6 \mathrm{~mm}$ & $100 \sim 300 \mathrm{~mm}$ \\
Thickness of projectile & $13.7 \mathrm{~mm}$ & $10 \sim 20 \mathrm{~mm}$ \\
Outer diameter of projectile & $123 \mathrm{~mm}$ & \\
Voltage & $5865 \mathrm{~V}$ & $3000 \sim 6000 \mathrm{~V}$ \\
Capacitance & $22.9 \mathrm{mF}$ & $1 \sim 31 \mathrm{mF}$ \\
Initial position of projectile & $25.9 \mathrm{~mm}$ & $0 \sim 100 \mathrm{~mm}$ \\
\hline
\end{tabular}

Because of the premature in optimal problem, the genetic algorithm for optimization is used. The genetic algorithm is based on evolution procedure through generations. The evolution consists of three steps: reproduction, crossover and mutation [10]-[11]. The optimal design results of the coilgun are shown in Table II.

The velocity, force as well as position of projectile and current flowing in driver coils simulated with the optimized parameters by FEA and the developed circuit model are shown in Figs. 15 to 18, respectively. The muzzle velocity simulated by the developed circuit model reaches $159 \mathrm{~m} / \mathrm{s}$ compared with the former result $86.82 \mathrm{~m} / \mathrm{s}$.

\section{CONCLUSION}

This paper presents the transient simulation and optimization for the capacitor-driven inductive coilgun. An improved projectile model is presented for accurate eddy current calculation. An equivalent circuit model is used to calculate the dynamic performance of coilgun. The simulated results are verified by FEA and experiment results. The influences of various design parameters on properties of induction electromagnetic launch system are analyzed. The optimization of coilgun is achieved by using genetic 
algorithm and the optimization results are in good agreement with FEA. By the comparisons between the optimized results and the original model, it can be concluded that the muzzle velocity can be increased. In the future work, the presented simulation and optimization method may be applied to the design of a multi-stage coilgun.

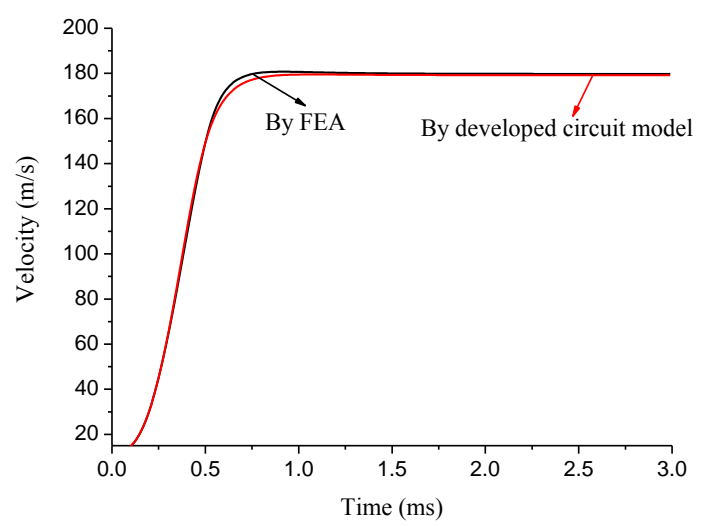

Fig. 15. Velocity based on optimized parameters

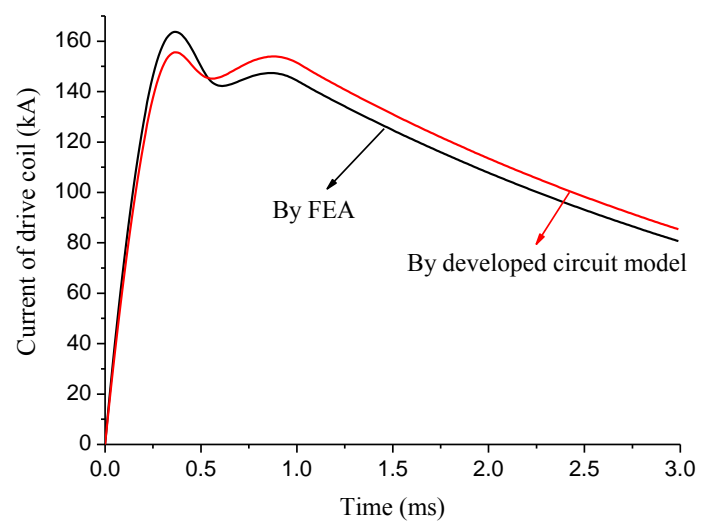

Fig. 16. Current of drive coil based on optimized parameters

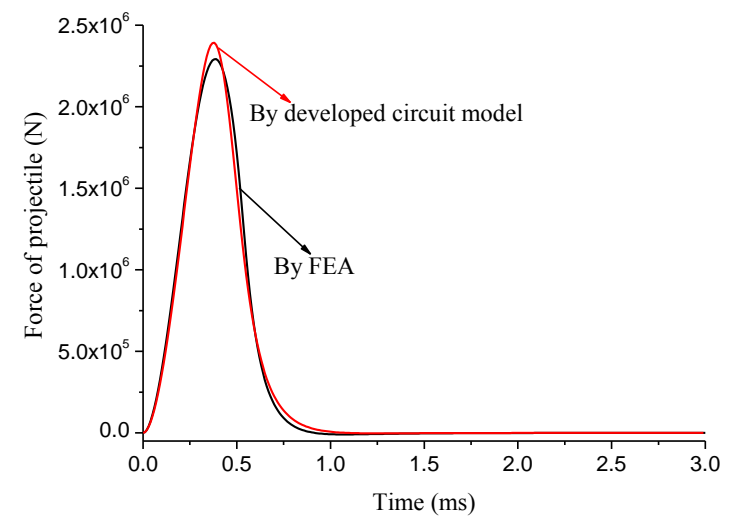

Fig. 17. Position of Projectile based on optimized parameters

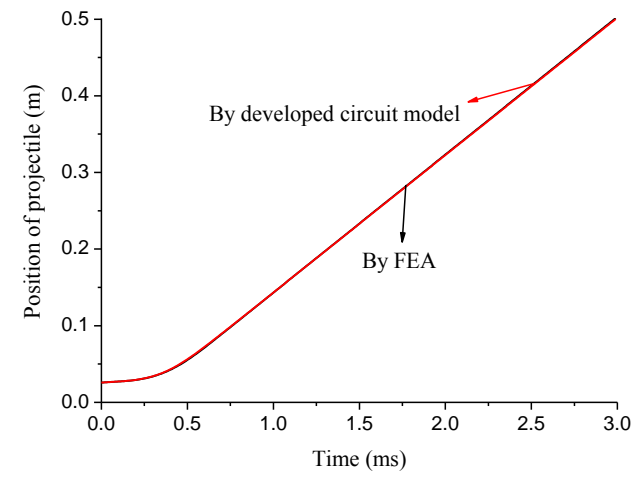

Fig. 18. Force of Projectile based on optimized parameters

\section{REFERENCES}

[1] H. Kolm and P. Mongeau, "Basic principles of coaxial launch technology," IEEE Trans. Magn., vol. 20, no. 2, pp. 227-230, 1984.

[2] M. Cowan, E. C. Cnare, B. W. Duggin, R. J. Kaye and T. J. Tucker, "The reconnection gun," IEEE Trans. Magn., vol. 22, no. 6, pp. 14291434, 1986.

[3] J. He, E. Levi, Z. Zabar, and L. Birenbaum, "Concerning the design of capacitively driven induction coil guns," IEEE Trans.Plasma Sci., vol. 17, no. 3, pp. 429-438, 1989.

[4] K. Mckinney and P. Mongeau, "Multiple stage pulsed induction acceleration," IEEE Trans. Magn., vol. 20, no. 2, pp. 239-242, 1984.

[5] Seog-Whan Kom, Hyun-Kyo Jung, and Song-Yop Hahn, "An optimal design of capacitor-driven coilgun," IEEE Trans. Magn., vol. 30, no. 2, pp. 207-211, 1994.

[6] T. J.Burgess, E. C. Cnare, W. L. Oberkampf, S. G. Beard and M. Cowan, "The electromagnetic $\theta$ gun and tubular projectiles," IEEE Trans. Magn., vol. 18, no. 1, pp. 46-59, 1982.

[7] Ronad J. Kaye, et al, "Design and performance of a multi-stage cylindrical reconnection launcher, "IEEE Trans. Magn., vol. 27, no. 1, pp. 596-600, 1991.

[8] Deman Wang, et al, "Coilgun (Electromagnetic coaxial launcher) and its systems analysis," Journal of Astronautics, vol. 17, no. 4, pp.79-82, 1996.

[9] Liyi Chen, Computer algorithms and applications, Tianjin University Press, 1990.

[10] D. E. Goldberg, Genetic Algorithms, Addison-Wesley, 1989.

[11] Ming Zhou, Shudong Sun,Genetic algorithms theiry and applications, National Defense Industry Press, 1996. 